\title{
Review puzzles and construction sets falling under the category of augmented reality games
}

\author{
Ilya $V$. Osipov ${ }^{1, *}$ and Evgeny Nikulchev ${ }^{2}$ \\ ${ }^{1}$ Study Inc, San Francisco, CA, USA \\ ${ }^{2}$ Moscow Technological University MIREA, 119454 Moscow, Russia
}

\begin{abstract}
In this article, the authors review puzzles and construction sets falling under the category of augmented reality games and, at the same time, serving as the elements of TUI interfaces. The review does not include games based on jigsaw puzzles (dissection puzzles, where the player has to put together a set of fragments of variously shaped images). Transreality puzzles are electronic-mechanical puzzles (usually three-dimensional), where the gameplay takes place both in the domain of a virtual game, and in that of a mechanical device.
\end{abstract}

\section{Introduction}

For almost forty years, the keyboard and the mouse have been the main means of the human-computer interaction. They are supplemented by the graphical user interface called the windows, icons, mouse, pointer (WIMP model) [1]. Almost all TUI projects contain some of the innovations listed below: touchscreen, accelerometer, computer mouse, computer vision, or various sensors. As they are adapted, simplified, and introduced into general practice, such interface devices and methods become mundane and start to be used in conventional graphical user interfaces.

The evolution of user interfaces started with the command-line interface invented in the 1960-70s. The first versions of the graphical interface (GUI) were developed at the Xerox Palo Alto Research Center in 1970. Now, GUIs are used everywhere in personal computers and mobile devices. User interfaces are developed further as a series of interconnected designs and concepts called conventionally tangible user interface, kinetic user interface, natural user interface, touch user interface, organic user interface, natural language user interface, kinetic user interface, gesture recognition, and even brain-computer interface.

The tangible user interface falls under the "tangible interaction" category. It is a variety of the user interface, in which a human being interacts with electronic devices by using material objects and structures [2].

The advantages of tangible interfaces were first recognized by manufacturers of video games, specifically, electronic arcade machines. Special TUIstyle devices were used in such machines already in the 1980s. Toy guns, steering wheels, pedals, joysticks provide much better interaction with video games. Additionally, game manufacturers started to build sensors into their products. For example, Boktai for
Game Boy Advance was equipped with an ultraviolet photo sensor, and real sunlight was required to charge the "weapon" in order to shoot vampires. Nintendo produced WarioWare: Twisted with a piezoelectric gyroscope. In addition to using buttons, players were supposed to shake, twist, and jiggle the console. Nonstandard controls imitating natural interaction with material subjects became a standard for Nintendo DS games: for example, in Zelda for DS, players should blow at the screen to propel the sails of windmills.

Gradually, TUI elements appear in unspecialized devices. Specifically, the iPhone interface is based partially on the principle of direct interaction with objects. "People do not understand that we invented a new class of interfaces," Steve Jobs said in 2007, and explained that "the matter is that the iPhone interface lacks verbs almost entirely." This peculiarity, which the head of Apple emphasized, is really very important. A user of the traditional graphical interface first chooses an object, and then chooses an action to perform from a menu (that very verb Jobs was talking about). A TUI user does not need a "verbal" menu: user picks an object and does what needs to be done. This is how multitouch gestures understood by the Apple phone work, it also works with browsing and map viewing in iPhones, as well as with inertial scrolling [3].

TUI techniques are also widely used in robotics, especially in those kinds of robots, which are designed to interact with humans [4].

In this article, the authors review puzzles and construction sets falling under the category of augmented reality games and, at the same time, serving as the elements of TUI interfaces. 


\section{Transreality puzzles, similar devices and construction sets}

1. Frazer's Intelligent Beermats and Three-dimensional Modelling System, were among the first cubes, where the physical shape was augmented by an electronic game. From the authors' viewpoint, they approach the mixed reality category, but do not enter it, since the virtual space of the games is weakly manifested. This is what Kelly Heaton, the Physical Pixels developer, tells about this project in her doctorate thesis: "John Frazer and his colleagues at the London Architectural Association have been innovating alternate representations of computation since the late 1960s. For thirty years, Frazer's research has been motivated by an "evolutionary architecture", or one in which the computer contributes generative rules, responsive behavior and other life-like qualities to building structures. Not surprisingly, he finds the twodimensionality of monitors and plotters restrictive for his exploration of a three-dimensional material space, and his background in architecture naturally leads him to physical modeling systems" [5].

TangledCube. Within the framework of this project, designers from the Faculty of Environment and Information Studies, Keio University in Japan created a Rubik's Cube, which serves as a computer interface and displays positions of its sides on a computer monitor in real time. The virtual space of the game is represented only on the display, which turns TangledCube into the interface of an entirely virtual reality game, rather than a self-sufficient instrument [6].

Triangles. This construction set was designed at the MIT Media Laboratory, shown in Fig. 5. It allows one to use triangles to build sophisticated shapes and review the results displayed on a computer monitor. The physical computer interface is a construction set consisting of identical plane plastic triangles, which are joined both physically and digitally, by means of magnetic conductive junctions. As in the previous case, the virtual space of the game is displayed on a computer monitor, which also makes Triangles an interface to an entirely virtual game, rather than a self-sufficient instrument [7].

ActiveCube. This project was developed at the Osaka University in Japan. Cubes can be assembled to form any shape, similar to LEGO. However, since each cube is equipped with a microprocessor and a parallel data transmission interface, the block of cubes "understands" the current configuration in real time. A computer receives the information about the collective set of blocks via the main block connector and shows the entire group on its display. If the operator moves blocks, the display shows the final configuration in real time. This project is similar to Triangles. The two projects, however, were designed by two design groups independently [8].

Peano is a set, which consists of fifty cubes, which, when joined, form a modular full-color 3D display. Each cube made of colorless diffuse plastic has an LED installed in the center. The LED illuminates the entire cube volume, and its color may vary. The cubes are combined in a modular network, which supports direct and software control of the structure color. Although the network topology is linear, its geometry is threedimensional, which is possible due to the use of the Peano curve. This curve (as well as its variant, the Hilbert curve) is a linear structure turning at an angle of 90 degrees to determine three-dimensional space in Cartesian coordinates. Magnetic mechanical connectors are designed to maintain connection in four directions and form a linear peer-to-peer network. Peano cubes can be animated by using a digital palette. Since the cubes are sensory and "are aware" of the network topology, they can be controlled by touching them [5].

Constructed Narratives. This construction set consists of 3D Pentomino tiles, which interact with each other and a PC via wireless channels and allow several people to play various games simultaneously. The PC displays show their virtual representation and additional information, e.g., colors and owners of the tiles, or letters for playing. According to the Jennings, the author of the project, Constructed Narratives is the first experiment in the field of studies of social tangible interfaces [9-10].

Siftables/Sifteo. In the authors' opinion, this is the first full-scale device falling under the transreality puzzles category. The elements of Siftables (Sifteo is the commercial name of this project) are both physical objects and virtual game elements at the same time. No external computers or displays are required. Thus, this is the first construction set or puzzle, which is a mixed reality object. Siftables elements look as toy bricks. The top of an element is a small-size LED display. Inside each tile, an accelerometer, four IR sensors (one per each side), and a microprocessor are installed. The tiles interact with each other and a PC. By means of the sensors, they "perceive" contacts with other tiles and can "feel", when they are lifted, shaken, or tilted. Test applications developed by the Siftables inventors at the MIT Media Laboratory demonstrate the familiar set of gestures used in tangible interfaces. A set of such tiles can be used as a platform for child's games [11-12].

Cubelets. The Cubelets game was developed by Modular Robotis. It is a toy set replaying the functionality of the ActiveCube and Triangles projects. However, its elements can be equipped with motorized units, as well as with sensors and other additional equipment. All this allows players to build toy robots, cars, and other items making the game more entertaining. From the authors' viewpoint, this project has few features in common with transreality puzzles. Rather, it is a computerized and motorized construction set and is presented here for illustration purposes only [13].

\section{Wawcube transreality puzzle}

The author (Osipov) has designed and made a prototype of an entirely "transreal" puzzle: a set called Wawcube. The main purpose of this puzzle is to join physical representation and virtual gaming environment in a common indivisible gameplay scenario [14].

According to Paul Milgram's concept of mixed reality, illustrated in Fig. 1, the proposed puzzle will fit in the middle of the scale, between augmented reality 
(AR) and augmented virtuality (AV), since the gameplay will require elements of the physical and virtual world equally. The gameplay lies at the boundary of the two media. Comparing it with the Siftables/Sifteo project, one can state that the latter is closer to augmented virtuality, since all Sifteo cubes can move around on a plane freely, and during the game, players focus mainly on what is happening in virtuality (see Fig. 1).
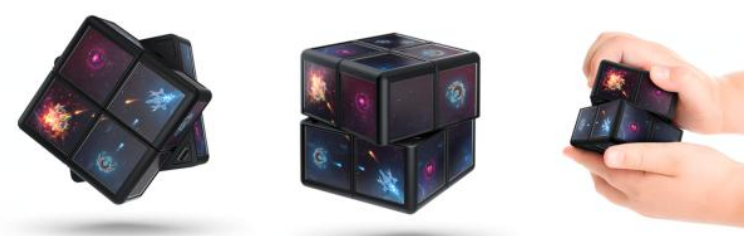

Fig. 1. Wawcube 3D-model (render).

Has been developed a prototype of the Wawcube set, shown in Fig. 2 and 3. The software was written in the $\mathrm{C}$ programming language, electronics was assembled on the basis of an ATmega328 and STM32 microprocessors, and bodies and electromagnetic connectors were printed on a 3D printer. In future versions, displays will occupy all possible space on side surfaces, and the frames around them, as well as the gaps between them will be minimized.

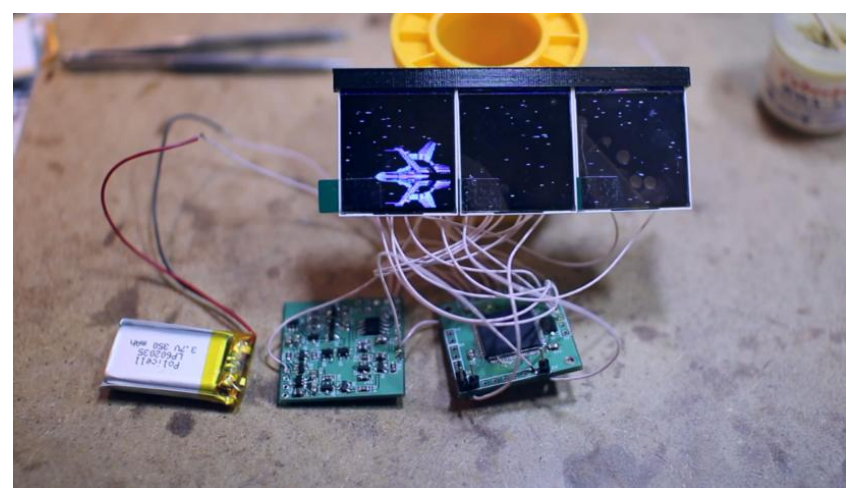

Fig. 2. The prototyping process and software testing

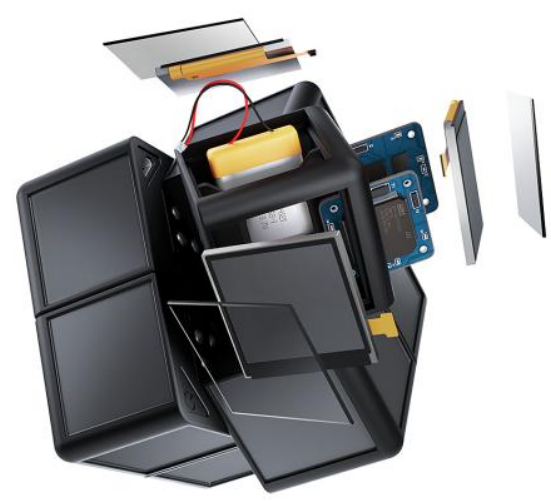

Fig. 3. Assembled and functional prototype Wawcube of 8-elements

\section{Discussion and conclusions}

The main question for such projects is how potential users will receive it. To find this out, it is planned to manufacture several full-scale prototypes, develop several full-scale games, and offer them to participants of test groups, who will play and study the device.

The test methods will be questionnaire surveys, test games, and contests (e.g., who will be the first to lead Pac-Man from one side to another without running into an obstacle, or who will be the first to make up ten words playing in the Scrabble regime). Test groups will include pre-school kids, schoolchildren, teenagers, as well as retired population. Testing by representatives of puzzle fan communities would be most welcome, as well as running tests with random adults in places, where people have to wait for a long times and would not object to being offered an interesting pastime, e.g., in airports. In addition, using the focus groups, it will be determined what construction is better for users, demountable or not. The tests will be finalized by questionnaire surveys, both direct and comparative ones (i.e., comparing the project with other puzzles or game consoles). The authors are planning to publish the results in a separate paper.

In conclusion, a review of mixed reality puzzles is conducted and the device called Wawcube, as an illustration of a mixed reality puzzle, is presented

\section{References}

1. R. J. Jacob, A. Girouard, L. M. Hirshfield et al. Reality-based interaction: unifying the new generation of interaction styles. In $\mathrm{CHI}^{\prime} 07$ extended abstracts on Human factors in computing systems, pp. 2465-2470 (2007)

2. O. Shaer, E. Hornecker,. Foundations and Trends in Human-Computer Interaction, 3, 1-137. (2010)

3. J. Markoff, As Apple Gains PC Market Share, Jobs Talks of a Decade of Upgrades (New York Times, 2002) http://www.nytimes.com/2007/10/22/ technology/22apple.html

4. C. Guo, E. Sharlin. Exploring the use of tangible user interfaces for human-robot interaction: a comparative study. In Proceedings of the SIGCHI Conference on Human Factors in Computing Systems, pp. 121-130 (2008)

5. K. B. Heaton, Physical pixels (Doctoral dissertation, Massachusetts Institute of Technology, 2000).

6. Y. Kamada, Y. Kakehi, TangledCube: A Proposal on an Electronic Puzzle Using a Rubik's Cube-typed Tangible Interface (Faculty of Environment and Information Studies, Keio University, 2010) http://www.interaction-ipsj.org/archives/paper2010/ demo/0140/0140.pdf

7. M. G. Gorbet, M. Orth, H. Ishii, Triangles: tangible interface for manipulation and exploration of digital information topography. In Proceedings of the SIGCHI conference on Human factors in computing 
systems, pp. 49-56 (ACM Press/Addison-Wesley Publishing Co., 1998)

8. Y. Kitamura, Y. Itoh, T. Masaki, F. Kishino. ActiveCube: a bi-directional user interface using cubes. In Proceedings. Fourth International Conference on Knowledge-Based Intelligent Engineering Systems and Allied Technologies, vol. 1, pp. 99-102 (IEEE, 2000)

9. P. Jennings, Tangible social interfaces: critical theory, boundary objects and interdisciplinary design methods. In Proceedings of the 5th conference on Creativity \& cognition, pp. 176-186 (ACM, 2005)

10. P. Jennings, A Theoretical Construct of Serious Play and the Design of a Tangible Social Interface. In Shared Encounters, pp. 153-172 (Springer, 2009)

11. S. Hunter, J. Kalanithi, D. Merrill, Make a Riddle and TeleStory: designing children's applications for the siftables platform. In Proceedings of the 9th International Conference on Interaction Design and Children, pp. 206-209 (ACM, 2010)

12. P. Milgram, H. Takemura, A. Utsumi, F. Kishino Augmented Reality: A class of displays on the reality-virtuality continuum, In SPIE 2351, Telemanipulator and Telepresence Technologies, pp. 282-292 (1995)

13. E. Schweikardt, Modular robotics studio. In Proceedings of the fifth international conference on Tangible, embedded, and embodied interaction, Portugal (2011)

14. I.V. Osipov, International Journal of Virtual and Augmented Reality, 1(2), 1-17 (2017) 